\title{
UAV PHOTOGRAMMETRY FOR FEATURE EXTRACTION AND MAPPING OF CORRUGATED INDUSTRIAL ROOFTOPS
}

\author{
Karun Reuel Dayal ${ }^{1}$, Inder Mohan Chauhan ${ }^{1}$ \\ ${ }^{1}$ Skylark Drones Pvt. Ltd. (karun,inder)@ skylarkdrones.com, karunrdayal@gmail.com, inderchauhan.esc@gmail.com
}

Commission IV, ICWG III/IVb and WG IV/4

KEY WORDS: photogrammetry, UAV, drones, solar panel, point cloud, corrugated roof

\begin{abstract}
:
Corrugated roof sheets used for large-scale industrial rooftops have the potential to carry solar panels. The sheets, however, need to be surveyed so that the solar panels can be installed according to the measurements of various features. For this purpose, information about exhausts, skylights, corrugation spacing, including extraction of roof slope, which is essential for planning solar panel installations, needs to be measured and mapped. UAV surveying, in contrast to traditional surveying and manual measurements, has proven to be of great benefit in the recent years due to its mobility and low-cost operations and with photogrammetric processing, 3D information with sufficient details can be obtained. In this study, we explored the use of UAV platforms for extraction of fine features (corrugations) of industrial rooftops. The point cloud obtained after photogrammetric processing contained significant geometric information about the corrugation, along with noise. The point cloud was processed to remove noise. Smoothing and exaggeration in the Z-direction, without altering $\mathrm{X}$ and $\mathrm{Y}$ coordinates, ensured a smooth profile with pronounced peaks at the location of corrugations. Cross-sectional profiles were extracted as a vector and peaks in the profile indicated presence of the corrugation. A peak identification algorithm was used to extract local maxima with corresponding distances along the profile. The peak points were plotted on the ortho-image, and the mean distance between subsequent peaks was approximately $20.3 \mathrm{~cm}$ with a standard deviation of $1-2 \mathrm{~cm}$. The spacing between the corrugations was approximately $20 \mathrm{~cm}$ when measured manually.
\end{abstract}

\section{INTRODUCTION}

Solar energy has the potential to meet our growing energy demands. Standard solar panels are easily installed on either the ground or flat concrete roofs. In such cases, the panels have standard dimensions, and a maximum possible number of such panels are fitted. Corrugated roof sheets used for large-scale industrial rooftops also have the potential to carry solar panels. The roof sheets, however, need to be surveyed so that the solar panels can be installed according to the measurements. For this purpose, information about exhausts, skylights, corrugation spacing, including extraction of roof slope, which is essential for planning solar panel installations, needs to be measured and mapped. The measurement is a manual process, which can be a laborious and time-consuming task, besides posing a significant risk to the safety of personnel. Further, the corrugated roof sheets can vary in dimensions and design and to manually measure all these variations may not be possible.

In the case of roof-shed corrugations, dimensions are in the range of few centimetres. Terrestrial laser scanners(TLS) have the potential to capture fine details (Vosselman and Maas, 2011). They are used in various applications such as geology (Buckley, Howell, Enge, and Kurz, 2008; Fowler and States, 2010; Giussani and Scaioni, 2004; Sturzenegger and Stead, 2009), cultural heritage (Briese, Pfeifer, and Haring, 2003; Camarda, Guarnieri, Milan, and Vettore, 2010; Grussenmeyer et al., 2011, 2012; Lerma, Navarro, Cabrelles, and Villaverde, 2010; Fabio Remondino, 2011), forestry (Liang et al., 2016; Richardson, Monika Moskal, and Bakker, 2014; White et al., 2016), structural health monitoring (Castellazzi, Altri, Bitelli, Selvaggi, and Lambertini, 2015; Guarnieri, Pirotti, Pontin, and Vettore, 2006; Olsen, Kuester, Chang, and Hutchinson, 2010; Turkan, Laflamme, and Tan, 2016), besides various other domains. The primary benefit of TLS that was utilized in these studies is the high-density, accurate sampling of 3D data over the target features. In structural health monitoring and building damage assessment, point spacing in the range of few centimetres is essential. In many of these studies, laser scanning was used in conjunction with close-range photogrammetry either to obtain high-quality texture information or for objectbased integration. Extraction of roof corrugation using TLS is possible with spacing in the range of few $\mathrm{mm}$ to $\mathrm{cm}$. With multiple scan positions, full roof mapping is possible. Notwithstanding the high-resolution measurements with a TLS, it comes at a significant cost and relatively low mobility.

In contrast to TLS, the combination of UAV platform and photogrammetry has proven to be of great benefit in the recent years due to its mobility and low-cost operations in various surveying requirements ranging from structural damage assessment to agriculture (Colomina, Blázquez, Molina, Parés, and Wis, 2008; Gnädinger and Schmidhalter, 2017; Lottes, Khanna, Pfeifer, Siegwart, and Stachniss, 2017; Ota, Ogawa, Mizoue, Fukumoto, and Yoshida, 2017; Persad and Armenakis, 2016; F Remondino, Barazzetti, Nex, Scaioni, and Sarazzi, 2011). The flying height can be controlled which results in flexibility in horizontal and vertical resolution of data, as per requirements. 3D information with sufficient details can be obtained using UAVs. To our knowledge, there is no specific study aimed at extracting rooftop corrugations using 3D point clouds. Zhang, (2006) mentions the use of UAV acquired images for studying condition of road surfaces. (Lovitt, Rahman, and Mcdermid, 2017) demonstrate the capability of UAV photogrammetry to map microtopographic terrain undulations subject to the complexity of the terrain. This study posed a unique challenge in terms of acquisition and technical requirements. On one hand, least possible flying height was necessary to resolve the vertical variations in roof corrugations and on the other, it was important to consider the capture of sufficient heterogeneous image content for successful calibration. Pix4D documentation highlights a horizontal accuracy of 1-2 times ground sampling distance (GSD) and a vertical accuracy of 1-3 times GSD. For a corrugation height of 
few centimetres, it was expected to achieve a sub-centimetre GSD which creates the possibility of some noise in the final data. A white paper by Pix4D on accuracy of UAV data documents a comparable accuracy with LiDAR. (Draeyer and February, 2014). The study tested the possibility of UAV data acquisition and processing for obtaining a sufficiently detailed point cloud and its subsequent processing to extract fine details.

\section{STUDY AREA AND DATA ACQUISITION}

The study was carried out on a corrugated roof shed in Bengaluru, India. The rooftops have homogenous texture which makes image calibration difficult. Acquisition had to be planned in a way to ensure the images calibrate, while retaining the geometric details of the roof by considering appropriate overlap between the consecutive images, flying height and viewing angle of the camera. For oblique acquisition, distance from the building is a major factor for GSD (resolution) of the data. Therefore, the distance was decided so to obtain the necessary geometric details of the roof corrugations. The following parameters were considered for the data acquisition:

- Vertical Flying height $=$ height of building +8 to $10 \mathrm{~m}$

- Horizontal Distance $=15 \mathrm{~m}$ from the wall .

- Overlap $=90 \%$

- Expected dataset properties

$$
\begin{array}{ll}
\circ & G S D=0.6 \mathrm{~cm} \\
\circ & \text { Image width }(\text { swath })=25 \mathrm{~m} \\
\circ & \text { Overlap }=22 \mathrm{~m}
\end{array}
$$

Figure 1 is a representation of the method of data acquisition that provided the desired results.

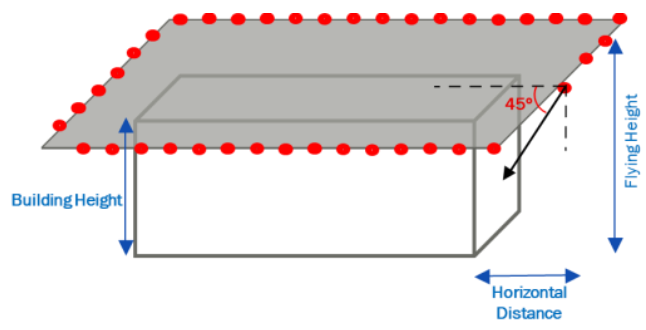

Figure 1: Data acquisition plan

Standard processing of data using Pix4D yielded the point cloud as shown in Figure 2. At this resolution, the 3D details of the roof were obtained, with some amount of noise. Figure $2 b$ is a zoomed-in view. The geometric properties of the roof are noticeable. The noise in the data set is visible in Figure 4.

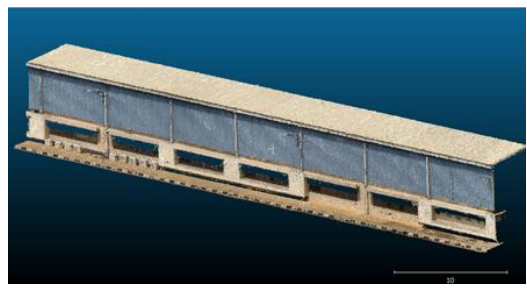

(a)

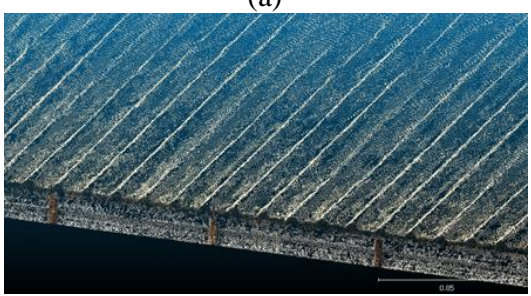

(b)

Figure 2: Output point cloud; a) Location, b) Magnified section

\section{METHODOLOGY}

This section describes the various steps involved in achieving the results. The primary objective of the study was to utilise the 3D information present in the point cloud, irrespective of the noise or inaccuracy, and modify it in a way that allowed for the automatic identification of locations of roof corrugations. The methodology describes the various tools and methods employed to process the point cloud. The initial photogrammetric processing is a standard practice adopted for generation of point clouds using UAV data.

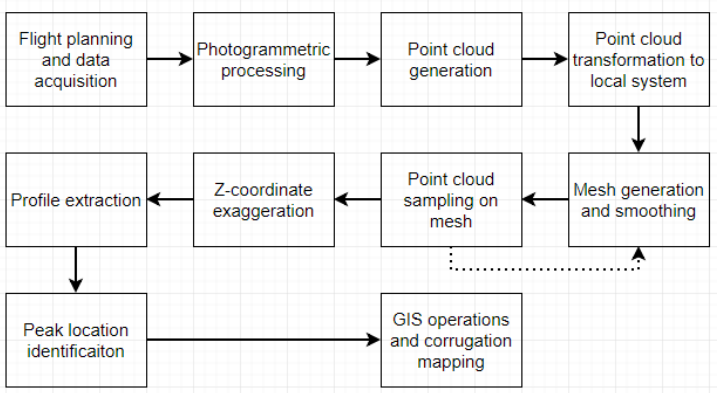

Figure 3: Methodological flow diagram

\subsection{Co-ordinate transformation}

The photogrammetric software generates point clouds in a local coordinate system or a georeferenced system based on the geotags of the raw images or the GCPs used for the purpose. In this study, the geo-tagged photos were used for processing, and the resulting point cloud was generated in UTM coordinates. UTM coordinates are large numbers and, therefore, the point cloud was translated to a local system. This prevented any loss in precision of the coordinates and reduced the multiplicative effect. The elevation of the region is around $800 \mathrm{~m}$. For example, for an elevation of 805 metres, multiplication with a factor of three will modify the elevation to $2415 \mathrm{~m}$. On translating by $-800 \mathrm{~m}$, the new coordinate in local system will be $5 \mathrm{~m}$. On multiplication by the same factor, it will be $15 \mathrm{~m}$. Translating back to original system will result in $815 \mathrm{~m}$, instead of $2415 \mathrm{~m}$ as in the former case.

\subsection{Point cloud pre-processing}

Photogrammetric processing depends on the image content. In relatively homogenous areas, there is considerable noise in the final point clouds, which is the case with a roof shed. Statistical Outlier Removal filter is useful for removal of sparse outliers. It was observed that filtering using the SOR tool resulted is loss of some information essential to identify the features. Instead of removing the noise points at the initial stage, a mesh was generated using all the points (including the noise points). Applying a Laplacian smoothing operator on the noisy mesh resulted in a surface that retained most of the original characteristics of the rooftop essential for the subsequent steps. This is illustrated in Figure 4. Points were then sampled on the smoothed mesh. 


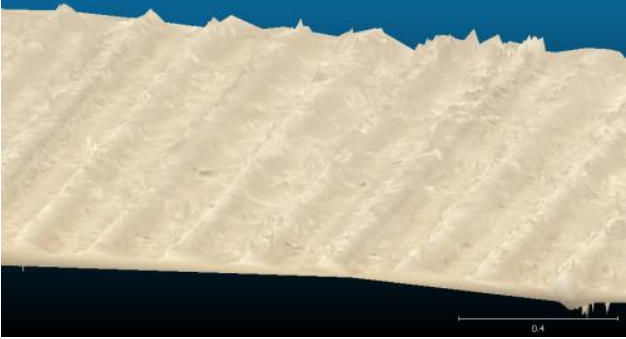

(a)

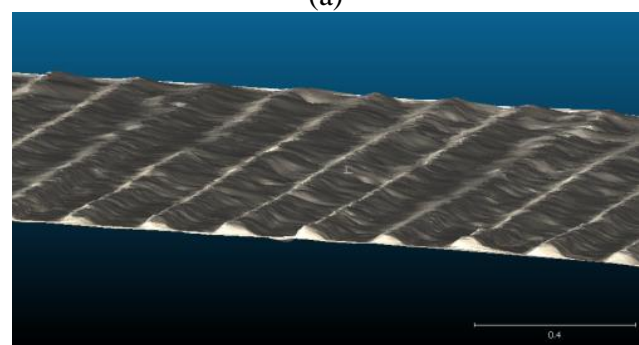

(b)

Figure 4: Mesh generation and smoothing; (a) Noisy mesh, (b) Smoothed mesh

\subsection{Z-coordinate exaggeration}

The photogrammetric point cloud obtained from the given height retained some of the geometric information to characterize the roof corrugation. However, due to the presence of significant noise, several smoothing operations resulted in a noiseless point cloud, sampled on the surface, with reduced geometric detail. The sampled point cloud however possessed variation in the $\mathrm{Z}$-direction. Smoothing operations reduced the variations that indicated the location of the corrugation. To enhance this information, the point cloud was exaggerated in the $\mathrm{Z}$-direction without affecting the $\mathrm{X}$ and $\mathrm{Y}$ directions. The $\mathrm{Z}$ coordinated was duplicated and converted to a scalar field that could be modified by arithmetic operations. The Z-coordinate, as a scalar field, was multiplied a suitable factor, in this case 3 . The existing Z-coordinate that defined the point cloud was replaced by the modified Z-coordinate resulting in a point cloud that was visibly exaggerated in the vertical direction, without affecting its $\mathrm{X}$ and $\mathrm{Y}$ values. Figure 6 shows the point cloud after exaggeration

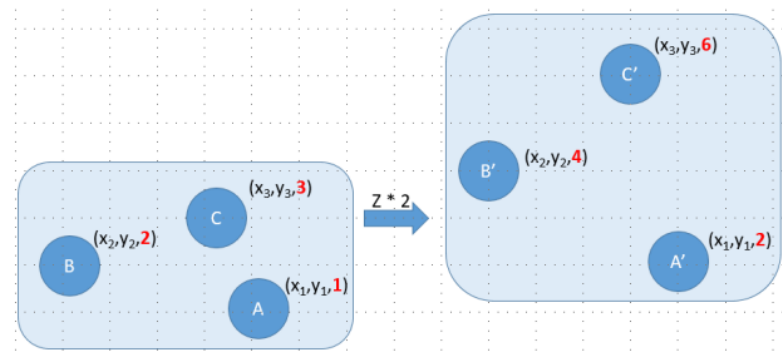

Figure 5: Exaggeration of point cloud

The exaggeration is depicted in Figure 5 where A,B,C are three points of a point cloud. The $\mathrm{Z}$ coordinate was multiplied by a factor of 2 .

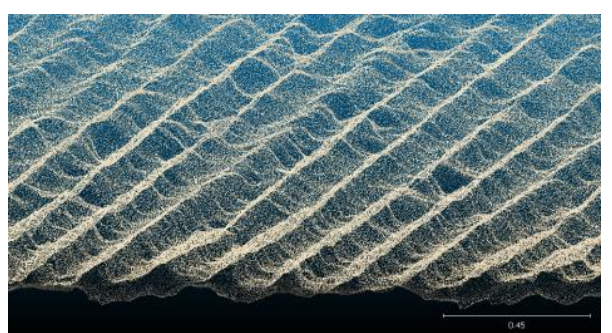

(a)

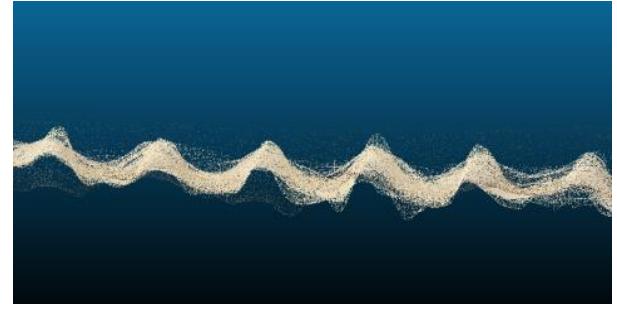

(b)

Figure 6: Smoothed and exaggerated point cloud

\subsection{Profile extraction and peak identification}

The exaggerated point cloud was converted to a mesh. Crosssectional profiles were extracted at high detail (at every change in elevation along the section). The profiles were obtained along parallel lines drawn on the mesh in the form of a vector. Signal processing tools were used to analyse the cross-section profile vector to find the local maxima. The local maxima approximately represented the location of the corrugations. It was essential that the vector had a smooth profile as flat peaks resulted in multiple local maxima. The toolbox identifies the local peaks and the distances at which they are present. The distances were used in linear referencing tools in ArcMap to create points at the specified locations along the original crosssectional line. The results are displayed in Figure 7.

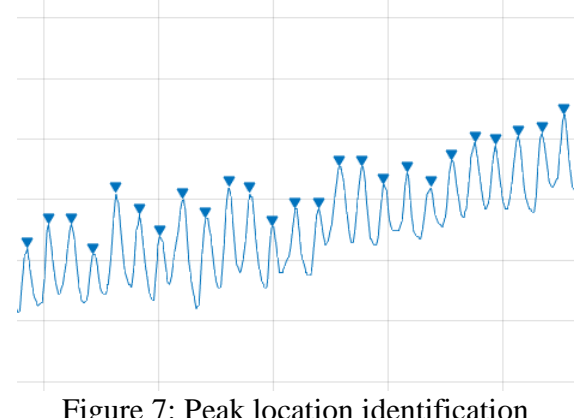

Figure 7: Peak location identification

\subsection{Best fit line and corrugation mapping}

The peak points of the profile were mapped on the orthomosaic/mesh of the rooftop. Every corrugation was represented by corresponding set of points. The points were not in a straight line as the different portion of the roofs had minor variations due to photogrammetric stitching, smoothing effects etc. The points were grouped according to a common ID (sequence of peaks along cross-sections) and averaging $\mathrm{XY}$ regression and YX regression lines resulted in an approximate best-fit line. We observed that the lines, which represent the corrugations, were restricted to the width of the same. 


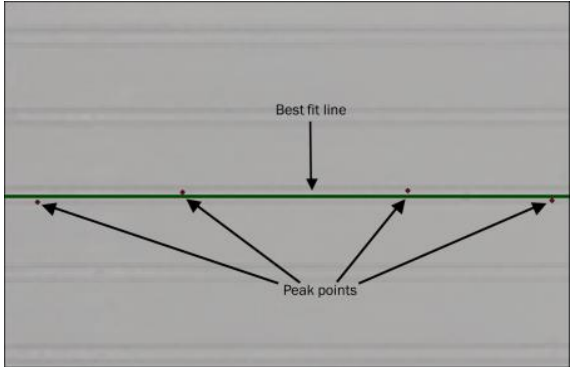

Figure 8: Peak points and corrugations

\section{RESULTS AND DISCUSSION}

Flying height played a crucial role in ensuring a point cloud with necessary geometric information was available to extract location and orientation of roof corrugations. A fine resolution allowed for the generation of a better point cloud in regions where the images had sufficiently heterogeneous content necessary for image calibration. In homogeneous areas, some images failed to calibrate thereby necessitating the UAV to be flown at different acquisition parameters. Some noise was also present which affected the accuracy of the profile. The roof corrugations were measured on field for inter-corrugation spacing. In one type of roof sheet, the inter-corrugation distance was around $20 \mathrm{~cm}$. In this methodology, the mean distance between subsequent peaks was approximately $20.3 \mathrm{~cm}$ with a standard deviation of $1-2 \mathrm{~cm}$.

Similarly, three other cross-sectional lines were analysed for peak locations and the standard deviation was in the range of 1$2 \mathrm{~cm}$. The average spacing of the peaks on respective crosssections were 20.2, 19.8 and 20.3.

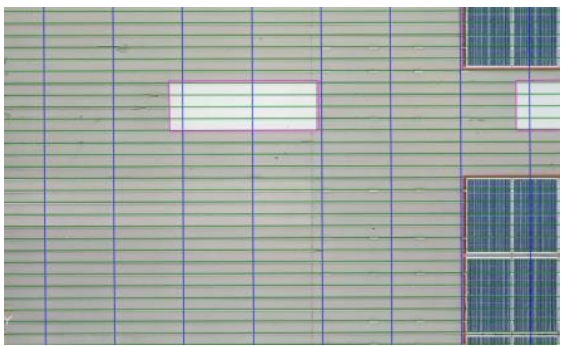

(a)

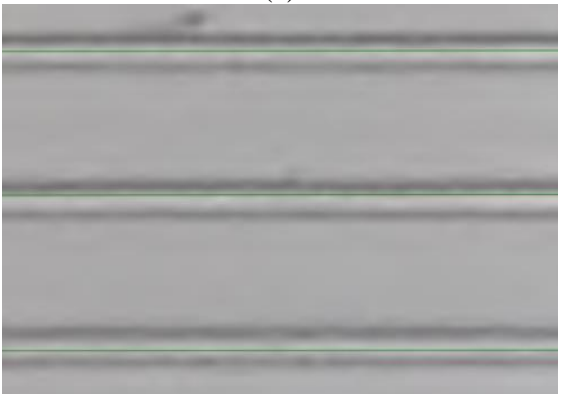

(b)

Figure 9: Mapped corrugations (green lines); (a) larger roof area, (b) magnified section

In Figure 9, the lines representing corrugations (green) are mapped on the ortho-image. Due to smoothing during generation of the mesh, the geometric details were lost and thereby resulting in smooth peaks in the locations of the corrugations. The margin of error in detection of the lines was limited to the width of the peaks, which in turn was limited to the width of the corrugations. This is apparent the Figure 7(b) where the three lines are overlaying at different positions across the width. This was observed across the entire roof. However, the number of lines were the same as the number of corrugations. Exaggeration of the point cloud ensured the locations of the corrugations were emphasized and this reduced any scope for missing them.

Images were acquired obliquely at the edge of the roof to ensure they possess sufficient heterogeneous content. Due to this, the images calibrated successfully and a point could be generated. The 3D information present in the point cloud, irrespective of the noise, was modified to obtain the desired results. This approach was suitable as opposed to image processing methods on ortho-images since they are highly dependent on the texture which, in turn, depends on the lighting conditions. Some acquisition plans resulted in washed out images thereby causing the photogrammetric process to fail. An increased flying height did not yield an ortho-image with sufficient resolution. On the field there is rarely liberty to control all parameters. Further study will focus on quantifying the acquisition parameters and the inter-relationship between GSD, flying height and vertical resolution of the outputs.

\section{CONCLUSIONS}

The focus of this study was limited to identification and extraction of corrugations on rooftops. Although some errors regarding the parallel condition of lines existed, these were rectified in the final CAD drawings. UAVs can be an effective tool in monitoring large-scale rooftops with minimal need for human involvement, which is essential for human safety as well. The large coverage offered by UAV data has the potential to complete the same task in a few days. Further study will focus on comprehensive coverage of entire roof to automate the extraction and mapping the corrugations along with other major details such as purlins, skylights, ventilators, etc.

\section{ACKNOWLEDGEMENTS}

The entire study was conducted at Skylark Drones Pvt. Ltd. The authors would like to express their gratitude to the organisation for supporting this study. The authors also thank all the team members for providing useful technical expertise when required.

\section{REFERENCES}

Briese, C., Pfeifer, N., and Haring, A., 2003. Laserscanning and Photogrammetry for the Modelling of The Statue Marc Anton, 3.

Buckley, S. J., Howell, J. A., Enge, H. D., and Kurz, T. H., 2008. Terrestrial laser scanning in geology: data acquisition, processing and accuracy considerations. Journal of the Geological Society, 165(3), 625-638. Retrieved from http://dx.doi.org/10.1144/0016-76492007-100

Camarda, M., Guarnieri, A., Milan, N., and Vettore, A., 2010. Health monitoring of complex structure using TLS and photogrammetry. International Archives of the Photogrammetry, Remote Sensing and Spatial Information Sciences - ISPRS Archives, 38(PART 5), 125-130. https://doi.org/10.1080/15583058.2011.606595

Castellazzi, G., Altri, A. M. D., Bitelli, G., Selvaggi, I., and Lambertini, A., 2015. From Laser Scanning to Finite Element 
Analysis of Complex Buildings by Using a Semi-Automatic Procedure. Sensors, 15(8), 18360-18380 https://doi.org/10.3390/s150818360

Colomina, I., Blázquez, M., Molina, P., Parés, M. E., and Wis, M., 2008. Towards a new paradigm for high-resolution low-cost photogrammetry and remote sensing. In The International Archives of the Photogrammetry, Remote Sensing and Spatial Information Sciences. Vol. XXXVII. Part B1. (pp. 1201-1206).

Draeyer, B., and February, C. S., 2014. White paper: How accurate are UAV surveying methods?

Fowler, A., and States, U., 2010. Applications of Advanced Laser Scanning Technology in Geology.

Giussani, A., and Scaioni, M., 2004. Application of TLS to support landslides study: survey planning, operational issues and data processing. International Archives of Photogrammetry, Remote Sensing and Spatial Information.

Gnädinger, F., and Schmidhalter, U., 2017. Digital counts of maize plants by Unmanned Aerial Vehicles (UAVs). Remote Sensing, 9(6). https://doi.org/10.3390/rs9060544

Grussenmeyer, P., Alby, E., Assali, P., Poitevin, V., Hullo, J.F., and Smigiel, E., 2011. Accurate documentation in cultural heritage by merging TLS and high-resolution photogrammetric data. In Proceedings of SPIE - The International Society for Optical Engineering (Vol. 8085, pp. 1-13). https://doi.org/10.1117/12.890087

Grussenmeyer, P., Alby, E., Landes, T., Koehl, M., Guillemin, S., Hullo, J. F., ... Smigiel, E., 2012. Recording approach of heritage sites based on merging point clouds from high resolution photogrammetry and Terrestrial Laser Scanning. In International Archives of the Photogrammetry, Remote Sensing and Spatial Information Sciences - ISPRS Archives (Vol. 39, pp. 553-558). https://doi.org/10.5194/isprsarchives-XXXIXB5-553-2012

Guarnieri, A., Pirotti, F., Pontin, M., and Vettore, A., 2006. 3D Surveying for Structural Analysis Applications. 3rd IAG / 12th FIG Symposium, 3(July), 22-24. Retrieved from http://www.fig.net/commission6/baden_2006/PDF/LS1/Guarnie ri.pdf

Lerma, J. L. J. L., Navarro, S., Cabrelles, M., and Villaverde, V. V., 2010. Terrestrial laser scanning and close range photogrammetry for 3D archaeological documentation: the Upper Palaeolithic Cave of Parpallo as a case study. Journal of Archaeological Science, 37(3), 499-507. https://doi.org/10.1016/j.jas.2009.10.011

Liang, X., Kankare, V., Hyyppä, J., Wang, Y., Kukko, A., Haggrén, H., ... Vastaranta, M., 2016. Terrestrial laser scanning in forest inventories. ISPRS Journal of Photogrammetry and Remote Sensing, $115, \quad 63-77$. https://doi.org/10.1016/j.isprsjprs.2016.01.006

Lottes, P., Khanna, R., Pfeifer, J., Siegwart, R., and Stachniss, C., 2017. UAV-Based Crop and Weed Classification for Smart Farming. In 2017 IEEE International Conference on Robotics and Automation (ICRA) (pp. 3024-3031). https://doi.org/10.1109/ICRA.2017.7989347
Lovitt, J., Rahman, M. M., and Mcdermid, G. J., 2017. Assessing the Value of UAV Photogrammetry for Characterizing Terrain in Complex Peatlands. Remote Sensing, 9. https://doi.org/10.3390/rs9070715

Olsen, M. J., Kuester, F., Chang, B. J., and Hutchinson, T. C., 2010. Terrestrial Laser Scanning-Based Structural Damage Assessment. Journal of Computing in Civil Engineering, 24(3), 264-272. 5487.0000028

Ota, T., Ogawa, M., Mizoue, N., Fukumoto, K., and Yoshida, S., 2017. Forest Structure Estimation from a UAV-Based Photogrammetric Point Cloud in Managed Temperate Coniferous Forests. Forests, 8, 1-11. https://doi.org/10.3390/f8090343

Persad, R. A., and Armenakis, C., 2016. Co-registration of DSMs generated by UAV and Terrestrial Laser Scanning Systems. In International Archives of the Photogrammetry, Remote Sensing and Spatial Information Sciences - ISPRS Archives (Vol. XLI-B1, pp. 985-990). https://doi.org/10.5194/isprsarchives-XLI-B1-985-2016

Remondino, F., 2011. Heritage recording and 3D modeling with photogrammetry and 3D scanning. Remote Sensing, 3(6), 11041138. https://doi.org/10.3390/rs3061104

Remondino, F., Barazzetti, L., Nex, F., Scaioni, M., and Sarazzi, D., 2011. UAV photogrammetry for mapping and 3D modeling - Current status and future perspectives -. In International Archives of the Photogrammetry, Remote Sensing and Spatial Information Sciences (Vol. XXXVIII, pp. 1-7).

Richardson, J. J., Monika Moskal, L., and Bakker, J. D., 2014. Terrestrial laser scanning for vegetation sampling. Sensors, 14(11), 20304-20319. https://doi.org/10.3390/s141120304

Sturzenegger, M., and Stead, D., 2009. Close-range terrestrial digital photogrammetry and terrestrial laser scanning for discontinuity characterization on rock cuts. Engineering Geology, 106(3-4), 163-182. https://doi.org/10.1016/j.enggeo.2009.03.004

Turkan, Y., Laflamme, S., and Tan, L., 2016. Terrestrial Laser Scanning-Based Bridge Structural Condition Assessment. Retrieved from http://lib.dr.iastate.edu/intrans_reports $\% 5 \mathrm{Cnhttp}: / /$ lib.dr.iastate. edu/intrans_reports/199

Vosselman, G., and Maas, H.-G., 2011. Airborne and terrestrial laser scanning. Whittles Publishing (Vol. 4). https://doi.org/10.1080/17538947.2011.553487

White, J. C., Coops, N. C., Wulder, M. A., Vastaranta, M., Hilker, T., and Tompalski, P., 2016. Remote Sensing Technologies for Enhancing Forest Inventories: A Review. Canadian Journal of Remote Sensing, 42(5), 619-641. https://doi.org/10.1080/07038992.2016.1207484

Zhang, C., 2008. An UAV-based Photogrammetric Mapping System. The International Archives of the Photogrammetry, Remote Sensing and Spatial Information Sciences. 